\title{
Continuous Positive Airway Pressure (CPAP) in the Treatment of Hypernasality
}

\author{
David P. Kuehn \\ Department of Speech and Hearing Science, University of Illinois at Urbana-Champaign, 901 S. Sixth Street, \\ Champaign, Illinois, 61820, USA.
}

\begin{abstract}
The purpose of this presentation is to provide a progress report and preliminary data pertaining to a study designed to test the efficacy of continuous positive airway pressure (CPAP) therapy in reducing hypernasality.
\end{abstract}

\section{INTRODUCTION}

Attempts to reduce hypernasality using therapeutic procedures has had a long and controversial history. A comprehensive review of this literature has been completed recently [5]. These authors point out that there appear to be three stages involved in this history. The first stage, approximately during the period of 1940 to the early 1960 s, was that of rather widespread acceptance of therapeutic attempts involving such procedures as blowing, swallowing, and sucking exercises. These procedures have not been shown to be effective and their usage led to a stage, approximately during the latter 1960s to early 1970s, in which many authorities were highly skeptical about whether therapeutic procedures could be effective in reducing hypernasality. More recently, since about the latter 1970s, there appears to be renewed hope that therapy may indeed be effective. This renewed hope is due, in part, to the availability and use of newer instrumentation such as nasoendoscopy which is capable of providing feedback to subjects during speech.

Feedback procedures eventually may be shown to be effective in reducing hypernasality, although data are needed. Such procedures presuppose that the individual being treated possesses the inherent anatomic and physiologic capability to modify his or her velopharyngeal activity to improve velopharyngeal functioning for speech. This view assumes that the individual can "learn" how to use the mechanism in a more appropriate manner thereby improving functioning for speech. However, it is possible that there are physical limitations that must be overcome before additional modification in behavior can be achieved. An example of such a limitation might be insufficient muscle strength or endurance coupled with a threshold of fatigue that is relatively low. An obvious approach to alleviate such a problem is to increase muscle strength or endurance using a resistance exercise regimen focused on the muscles of the soft palate.

Kuehn [1] proposed a resistance type of treatment for strengthening the muscles of velopharyngeal closure that utilizes well-known principles of exercise physiology. Resistance to the muscles is provided by delivering heightened air pressure to the nasal cavities using a commercially available device referred to generically as continuous positive airway pressure (CPAP). Major principles of the treatment include overloading, progressive resistance, and specificity of the exercise regimen, all of which are known to increase muscle strength. Overloading refers to the phenomenon in which muscles adapt to the load to which they are subjected. The greater the overload, the greater the strength gain that can be expected for the muscle or muscle group being exercised. Progressive resistance refers to the gradual increase in the "intensity" of the exercise program, such as increasing the weight of an object lifted. Such an increase is necessary as muscles adapt to a higher load bearing capacity. If overloading does not occur, further increases in strength will not be obtained. Specificity involves exercising the particular muscles and, presumably, activating the corresponding motor nerve pathways that will be used during a given task. This is an important consideration with regard to the muscles of velopharyngeal closure because nonspeech activities, such as swallowing and blowing, still are frequently used in an attempt to strengthen the muscles. However, it is known that nonspeech tasks, such as swallowing, involve very different velopharyngeal movement patterns compared to that for speech. Thus, it is advantageous to provide resistance to the muscles of velopharyngeal closure during speech.

The muscles of velopharyngeal closure appear to be vulnerable to fatigue because velar elevation during speech is a repetitive activity that may occur frequently within a relatively short amount of time. For individuals with a normal mechanism, levator veli palatini muscle activity for speech occurs at a fairly low level within its total operating range [2] and thus is less susceptible to the effects of fatigue. However, for individuals with surgically repaired cleft palate, levator activity for speech occurs at a relatively high level within its total operating range [3] and, presumably, is much more susceptible to the effects of fatigue. Individuals with marginal velopharyngeal inadequacy, in particular, may tend to elevate the velum to a position involving muscle activity that is just below the muscles' threshold of fatigue. This would tend to avoid a situation in which fatigue occurs and velar elevation becomes very difficult or impossible resulting in very severe hypernasality. Unfortunately, the prevailing velar position achieved may not be sufficient for tight velopharyngeal closure and some degree of hypernasality might result. If fatigue effects are at issue, it appears that individuals with velopharyngeal inadequacy for speech would benefit from exercises that increase muscle endurance as well as strength. Endurance refers to a muscle's ability to maintain a constant tension over prolonged or repeated contraction. The CPAP therapy proposed by Kuehn [1] 
could potentially increase both muscle endurance as well as strength because it overloads the levator veli palatini muscle [4] and involves a regimen with a large number of repetitions of velar elevation.

\section{METHOD}

\subsection{Cooperating Centers}

Several Cleft Palate Centers have cooperated in this research project. Originally, the following investigators contributed: 1) David L. Jones, Riley Hospital for Children, Indianapolis, 2) Mary M. O'Gara, Northwestern University, Chicago, 3) Bonnie E. Smith, University of Illinois at Chicago, 4) Duane R. VanDemark, University of Iowa, Iowa City, 5) Jayne M. Wachtel, Carle Clinic Association, Urbana, Illinois, 6) Michael P. Karnell, University of Chicago, and 7) Lynn M. Grames, St. Louis Children's Hospital. Because of relocation of personnel and other factors, Investigators Karnell and Grames are no longer participating and Earl Seaver, Ph.D., Northern Illinois University and Rockford Illinois Cleft Palate Clinic has been added.

\subsection{CPAP Procedure}

The continuous positive airway pressure (CPAP) instrumentation consists of an air pressure generating source and a hose and mask assembly. The mask is placed over the nose of the subject and the increased air pressure is delivered directly to the nasal cavities. Specially constructed speech utterances of the form VNCV, such as /insi/, are used for drillwork. These nonsense words are designed to lower the velum during the nasal consonant $(\mathrm{N})$ followed by a rigorous elevation of the velum for the following pressure consonant (C) much like the power lift in weight training. The heightened air pressure in the nasal cavities provide the "weight" against which the muscles of velopharyngeal closure must work. Fifty such words are produced in sequence followed by six short sentences to generalize more closely to conversational speech. Similar sets are repeated until the session time expires.

Therapy sessions are conducted in the subject's home and involve one session per day, six sessions per week, over a total duration of eight weeks. The time of each session progresses from 10 minutes during the first week, 12 minutes during the second week, etc. up to 24 minutes during the eighth week. The air pressure delivered to the nasal cavities also increases in accordance with the principle of progressive resistance exercise such that the lowest pressure is $4.0 \mathrm{~cm} \mathrm{H}_{2} \mathrm{O}$ during the first week and the highest pressure of $8.5 \mathrm{~cm} \mathrm{H}_{2} \mathrm{O}$ is reached during the eighth week. follow the same protocol. Subjects are seen over four visits. They receive instruction on CPAP machine usage and therapy procedure during the first visit. Also during the first visit, the following are performed: 1) high quality audio tape recording, 2) nasometry using the Kay Elemetrics Nasometer providing "nasalance" measures, and 3) frontal- and lateral-view videofluoroscopy. These activities are repeated during the second, third, and fourth visits except that videofluoroscopy is omitted during the second visit. The second and third visits are spaced four weeks apart relative to the first visit. The fourth visit occurs one year after the third visit as a follow-up evaluation.

As in any treatment efficacy study, compliance is an important factor. In an attempt to encourage compliance and to measure it, subjects and their care providers are required to tape record each therapy session in its entirety. Subsequently, these recordings are evaluated to determine whether in fact subjects completed each session according to the time designated. Irregularities, such as unusually long breaks, are noted.

\section{$2.4 \quad$ Outcome Measures}

The audio recordings from each visit, the nasalance values, and measures obtained from the x-ray videos are being used to provide outcome data. With regard to the audio recordings, reference tapes are being prepared to serve as references against which experienced listeners will compare subjects' recordings without knowledge of which visit the recordings were obtained. There are three separate reference tapes, adult male, adult female, and prepubertal male and female. Each reference tape consists of eight samples arranged along an equal-appearing-interval scale from normal to the most severely hypernasal. The reference tapes are being carefully prepared using an iterative listening procedure in which several experienced listeners use a visual scale to indicate the proximity of one sample to adjacent samples. If the perceptual proximity of any two samples is too close (less than a distance of 25\%) then that sample or samples are discarded and replaced until the proper perceptual spacing is achieved.

As of this writing, nasalance data were available for 34 subjects. Of these 34 , nasalance scores were lower (less nasal) for 23 subjects during the third visit, following eight weeks of therapy, compared to nasalance scores obtained during the first visit. It is anticipated that nasalance analysis will be complete, with the exception of the one-year follow-up data for more recently enrolled subjects, at the time of the ICSLP 96 conference.

\subsection{Protocol}

All six centers currently participating in the project 


\section{REFERENCES}

1. Kuehn, D.P. (1991). New therapy for treating hypernasal speech using continuous positive airway pressure (CPAP). Plastic \& Reconstructive Surgery, $88,959-966$.

2. Kuehn, D.P. \& Moon, J.B. (1994). Levator veli palatini muscle activity in relation to intraoral air pressure variation. Journal of Speech and Hearing Research 37, 1260-1270.

3. Kuehn, D.K. \& Moon, J.B. (1995). Levator veli palatini muscle activity in relation to intraoral air pressure variation in cleft palate subjects. Cleft PalateCraniofacial Journal,32,376-381.

4. Kuehn, D.P., Moon, J.B., \& Folkins, J.W. (1993).

Levator veli palatini muscle activity in relation to intranasal air pressure variation. Cleft PalateCraniofacial Journal,30361-368.

5. Tomes, L., Kuehn, D.P., \& Peterson-Falzone, S. (in press). Behavioral therapy for speakers with velopharyngeal impairment. In K.R. Bzoch (Ed.), Communicative Disorders Related to Cleft Lip and Palate, 4th Ed, Austin, TX: Pro-Ed. 